\title{
Pre-clinical evaluation of eight DOTA coupled gastrin-releasing peptide receptor (GRP-R) ligands for in vivo targeting of receptor-expressing tumors
}

Antonella Accardo ${ }^{1}$, Filippo Galli ${ }^{2}$, Rosalba Mansi ${ }^{3}$, Luigi Del Pozzo ${ }^{3}$, Michela Aurilio ${ }^{4}$, Anna Morisco ${ }^{4}$, Paola Ringhieri ${ }^{1}$, Alberto Signore ${ }^{2}$, Giancarlo Morelli ${ }^{1}$ and Luigi Aloj ${ }^{5^{*}}$

\begin{abstract}
Background: Overexpression of the gastrin-releasing peptide receptor (GRP-R) has been documented in several human neoplasms such as breast, prostate, and ovarian cancer. There is growing interest in developing radiolabeled peptide-based ligands toward these receptors for the purpose of in vivo imaging and radionuclide therapy of GRPR-overexpressing tumors. A number of different peptide sequences, isotopes, and labeling methods have been proposed for this purpose. The aim of this work is to perform a direct side-by-side comparison of different GRP-R binding peptides utilizing a single labeling strategy to identify the most suitable peptide sequence.

Methods: Solid-phase synthesis of eight derivatives (BN1-8) designed based on literature analysis was carried out. Peptides were coupled to the DOTA chelator through a PEG4 spacer at the N-terminus. Derivatives were characterized for serum stability, binding affinity on PC-3 human prostate cancer cells, biodistribution in tumorbearing mice, and gamma camera imaging at 1, 6, and $24 \mathrm{~h}$ after injection.

Results: Serum stability was quite variable among the different compounds with half-lives ranging from 16 to $400 \mathrm{~min}$ at $37^{\circ} \mathrm{C}$. All compounds tested showed $K_{\mathrm{d}}$ values in the nanomolar range with the exception of BN3 that showed no binding. Biodistribution and imaging studies carried out for compounds BN1, BN4, BN7, and BN8 showed targeting of the GRP-R-positive tumors and the pancreas. The BN8 compound (DOTA-PEG-DPhe-GIn-TrpAla-Val-NMeGly-His-Sta-Leu- $\mathrm{NH}_{2}$ ) showed high affinity, the longest serum stability, and the highest target-tobackground ratios in biodistribution and imaging experiments among the compounds tested.

Conclusions: Our results indicate that the NMeGly for Gly substitution and the Sta-Leu substitution at the Cterminus confer high serum stability while maintaining high receptor affinity, resulting in biodistribution properties that outperform those of the other peptides.
\end{abstract}

Keywords: Gastrin-releasing peptide receptor, Radiolabeled peptides, Prostate cancer, Biodistribution

\footnotetext{
* Correspondence: l.aloj@istitutotumori.na.it

${ }^{5}$ Struttura Complessa Medicina Nucleare, Istituto Nazionale Tumori

"Fondazione G. Pascale"-IRCCS, Via M. Semmola, 52, Napoli 80131, Italy

Full list of author information is available at the end of the article
} 


\section{Background}

The bombesin receptor family consists of four receptor subtypes (BB1, BB2/GRP, BB3, and BB4) [1]. Overexpression of the first two members of this family has been documented in several human neoplasms such as breast, prostate, and ovarian cancer and is of interest for diagnostic and therapeutic nuclear medicine applications [2]. In patients with prostate cancer, there is very promising preliminary clinical data pointing to the use of radiolabeled gastrin-releasing peptide receptor (GRP-R) ligands for detecting metastatic disease and monitoring disease progression $[3,4]$. There is also great interest in developing peptide receptor radionuclide therapy (PRRT) strategies with the same approach given the high uptake values found in metastatic lesions. Initially, the GRP-R agonist AMBA [5] showed promising results for successful clinical use; however, its agonistic activity has caused acute gastrointestinal side effects such as nausea, abdominal pain, and emesis and its overall efficacy has been rather unsatisfactory in a phase I trial [6]. Later work has focused on the development of receptor antagonists that have the advantage of showing higher specific uptake compared to agonists and none of the gastrointestinal side effects shown by agonists [7]. Efforts are made to identify optimal ligands that, labeled with appropriated radionuclides (e.g., ${ }^{68} \mathrm{Ga}$ for PET imaging and ${ }^{177} \mathrm{Lu}$ for therapy), can be used for imaging and therapy applications in a "theranostic" approach.

Several radiolabeled bombesin agonists and antagonists have been described in recent years where numerous peptide sequences and chelation systems have been extensively studied. Optimization of different labeling strategies and modification of the peptide structures aimed at improving stability, pharmacokinetic, and binding properties of the peptides have been addressed. Most of the derivatives are based on the peptide sequence of truncated bombesin, bombesin (7-14) (BN(7-14): GlnTrp-Ala-Val-Gly-His-Leu-Met- $\mathrm{NH}_{2}$ ), that retains high affinity for the GRP-R [8].

Modifications in key positions of $\mathrm{BN}(7-14)$ are crucial not only to stabilize the derivatives or to increase the binding affinity but also in determining whether these molecules have agonist or antagonist properties. A comprehensive summary of these studies has been reported by Jensen et al. [9]. The methionine on the C-terminus $\left(\mathrm{Met}^{14}\right)$ is sensitive to oxidation and has been substituted with norleucine (Nle) [10-12]. The replacement of Leu ${ }^{13}$ with $\mathrm{Cha}^{13}$ has shown to improve overall stability and in vivo targeting ability of the peptide [13]. The $\mathrm{His}^{12} /$ $\mathrm{Leu}^{13}$ bond is sensitive to cleavage by neutral endopeptidase [14], so substitution of Leu for the non-natural Cha and Sta (see below) has been suggested to increase in vivo stability [15]. The Gly ${ }^{11}-\mathrm{His}^{12}$ bond is sensitive to carnosinase enzymatic cleavage [16] and therefore Gly ${ }^{11}$ has been substituted with $N$-methylglycine in previous reports [15]. Finally, the presence of $D P h e$ at the Nterminus and the substitution of the Leu ${ }^{13}$ with the unnatural aminoacid $\mathrm{Sta}^{13}$ seem to confer antagonistic behavior to the new analogs [17]. Bombesin receptor antagonists are shown to be superior to agonists in terms of higher tumor accumulation, longer tumor retention, and better tumor-to-tissue ratios $[7,18,19]$.

The introduction of a spacer between the chelator and the peptide is an important feature since it can modulate the stability, affinity, and pharmacokinetics of the bombesin derivatives. It has been shown that polyethylene glycol moieties of different lengths improve pharmacokinetics of bombesin derivatives [20-23], although they may negatively affect affinity and internalization rates $[23,24]$.

All of the above described modifications have been previously described using different labeling strategies and methods to characterize in vitro and in vivo biological properties. Our current goal is to investigate the importance of the peptide sequence in determining overall biological properties of the previously described compounds. To achieve this, we have synthesized and characterized eight different derivatives that have been coupled at the $\mathrm{N}$-terminus with the DOTA chelator through a PEG4 (15-amino-4,7,10,13-tetraoxapentadecanoic acid) spacer in order to use the same labeling strategy for all. We have performed in vitro and in vivo experiments (such as stability, binding affinity, biodistribution, and tumor targeting) to obtain a side-by-side comparison of these compounds to determine which of these peptide sequences is more suitable for in vivo use.

\section{Methods}

\section{Reagents}

Protected $\mathrm{N}^{\alpha}$-Fmoc-amino acid derivatives, coupling reagents, and Rink amide 4-methylbenzhydrylamine (MBHA) resin were purchased from CalbiochemNovabiochem (Laufelfingen, Switzerland). The Fmoc-21amino-4,7,10,13,16,19-hexaoxaheneicosanoic acid (Fmoc -Ahoh-OH) and Fmoc-8-amino-3,6-dioxaoctanoic acid (Fmoc-AdOO-OH) were purchased from Neosystem (Strasbourg, France). DOTA(OtBu) $)_{3}-\mathrm{OH}$ (1,4,7,10-tetraazacyclododecane-1,4,7,10-tetraacetate tert-butyl ester) was purchased from CheMatech (Dijon, France). Citrate acid, sodium citrate, and sodium chloride were obtained from Sigma-Aldrich Corp. (St. Louis, MO, USA). All other chemicals were commercially available by SigmaAldrich, Fluka (Bucks, Switzerland) or LabScan (Stillorgan, Dublin, Ireland) and were used as received unless otherwise stated. Preparative HPLCs were carried out on a LC8 Shimadzu HPLC system (Shimadzu Corporation, Kyoto, Japan) equipped with a UV Lambda-Max Model 481 detector. UV-Vis measurements were carried out on Thermo Fisher 
Scientific Inc. (Wilmington, Delaware USA) Nanodrop 2000c spectrophotometer equipped with a $1.0-\mathrm{cm}$ quartz cuvette (Hellma). ${ }^{177} \mathrm{LuCl}_{3}$ and ${ }^{111} \mathrm{InCl}_{3}$ were obtained from IDB (Petten, The Netherlands) and Mallinckrodt Radiopharmaceuticals Italia (Segrate, Italy), respectively.

\section{DOTA-PEG4-BN peptide syntheses}

All peptide conjugates were synthesized according to the standard solid-phase techniques with $\mathrm{Fmoc} / \mathrm{tBu}$ protocols using a Syro I MultiSynThec GmbH (Wullener, Germany) automatic synthesizer. Rink-amide MBHA resin $(0.78 \mathrm{mmol} / \mathrm{g}, 0.5 \mathrm{mmol}$ scale, $0.640 \mathrm{~g})$ was used. Elongation of the BN analogs was achieved by serial addition of 4 equivalents of Fmoc-AA-OH, 4 equivalents of the coupling reagents $\mathrm{PyBOP} / \mathrm{HOBt}$ (benzotriazol-1yl-oxytripyrrolidinophosphonium hexafluorophosphate/ 1-hydroxy-1,2,3-benzotriazole), and 8 equivalents of $\mathrm{N}, \mathrm{N}$ diisopropylethylamine (DIPEA) (1:1:2) in dimethylformamide (DMF). Each coupling was performed twice leaving the mixture under stirring for $30 \mathrm{~min}$. Deprotection of the Fmoc group from the N-terminus of the sequence was achieved with 2 cycles $(7 \mathrm{~min}$ each) of a piperidine solution in DMF $(70 / 30 v / v)$. When the bombesin sequences were complete, and the last Fmoc N-terminal protecting group removed, two residues of Fmoc-AdOO-OH and the DOTA $(\mathrm{OtBu}) 3-\mathrm{OH}$ chelating agent were condensed as previously described [25]. For the deprotection and cleavage, the peptidyl resins were treated with trifluoroacetic acid (TFA) containing $2.5 \%$ triisopropylsilane (TIS) $(v / v)$ and $2.5 \%$ water $(v / v)$ as scavengers for $2 \mathrm{~h}$. Then, crude peptide conjugates were precipitated at $0{ }^{\circ} \mathrm{C}$ by adding cold ether dropwise and lyophilized. Purification was
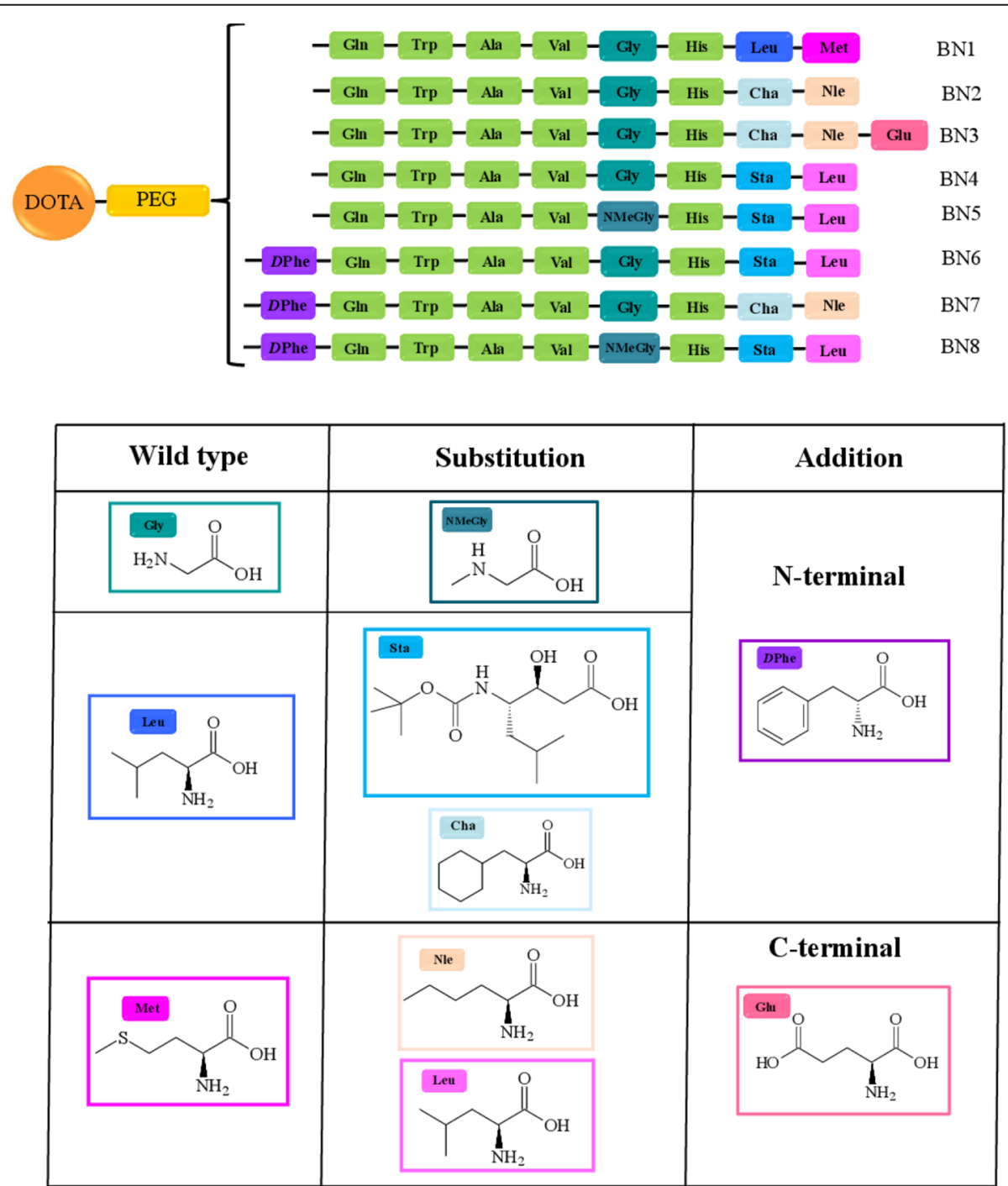

Fig. 1 Schematic representation of BN peptide conjugates DOTA-PEG4-BN. BN sequences are here indicated using the three-letter amino acid code. Substitutions with respect to the wild-type BN[7-14] and addition at the C- or N-terminus are also shown 
carried out by RP-HPLC $(\lambda=280 \mathrm{~nm})$ on a LC8 Shimadzu HPLC system (Shimadzu Corporation, Kyoto, Japan) equipped with a UV Lambda-Max Model 481 detector using a Phenomenex (Torrance, CA) C18 (300 ̊, $250 \times$ $21.20 \mathrm{~mm}, 5 \mu$ ) column eluted with $\mathrm{H}_{2} \mathrm{O} / 0.1 \%$ TFA (A) and $\mathrm{CH}_{3} \mathrm{CN} / 0.1 \%$ TFA (B) from 5 to $70 \%$ over $30 \mathrm{~min}$ at a flow rate of $20 \mathrm{~mL} \mathrm{~min}^{-1}$. Figure 1 shows a schematic representation of the different peptide sequences synthesized and highlights how they have been modified from the wild-type bombesin octapeptide BN[7-14] (BN1). Purity and identity of the products (see Table 1) were assessed by LC-MS analyses using Finnigan Surveyor MSQ single quadrupole electrospray ionization (Finnigan/ Thermo Electron Corporation, San Jose, CA) equipped with a C18-Phenomenex column $(250 \times 4.6 \mathrm{~mm})$. Each peptide solution was eluted with $\mathrm{H}_{2} \mathrm{O} / 0.1 \%$ TFA (A) and $\mathrm{CH}_{3} \mathrm{CN} / 0.1 \%$ TFA (B) from 5 to $70 \%$ over $15 \mathrm{~min}$ at $250 \mu \mathrm{L} \mathrm{min}{ }^{-1}$ flow rate. The yields of the peptides after the purification ranged between 60 and $80 \%$ and purity degree, obtained by integrating the HPLC areas, higher than $97 \%$.

\section{Serum stability}

Serum stability experiments were carried out on ${ }^{177} \mathrm{Lu}-$ labeled peptides due to weekly availability of this isotope in the laboratory where the experiments were performed. All the ${ }^{177} \mathrm{Lu}$-labeled conjugates were obtained dissolving $10 \mu \mathrm{g}$ peptide in ammonium acetate buffer $(300 \mu \mathrm{l}, 0.4 \mathrm{M}, \mathrm{pH} 5.5)$ and incubating for $30 \mathrm{~min}$ at $95^{\circ}$ $\mathrm{C}$ with ${ }^{177} \mathrm{LuCl}_{3}$ (100-180 MBq). Quality controls to determine the labeling yield and the radiochemical purity were performed using radio-HPLC. Radiolabeled peptides were utilized without further purification. To $1 \mathrm{~mL}$ of freshly prepared human serum, previously equilibrated in a $5 \% \mathrm{CO}_{2}$ environment at $37{ }^{\circ} \mathrm{C}$, we added $0.6 \mathrm{nmol}$ of the ${ }^{177} \mathrm{Lu}$-labeled peptide. The mixture was incubated in a $5 \% \mathrm{CO}_{2}, 37{ }^{\circ} \mathrm{C}$. At different time points, $100 \mu \mathrm{L}$ aliquots (in triplicates) were removed and treated with $200 \mu \mathrm{L}$ of $\mathrm{EtOH}$ to precipitate serum proteins. Samples were then centrifuged for $15 \mathrm{~min}$ at $5000 \mathrm{rpm}$ (2655g). After centrifugation, $50 \mu \mathrm{L}$ of supernatant was removed and counted in a $\gamma$-well counter; the sediment was washed twice with $1 \mathrm{~mL}$ of EtOH and counted. The activity in the supernatant was compared with the activity in the pellet to obtain the percentage of peptide not bound to proteins or radiometal transferred to serum proteins. The supernatant was also analyzed with radioHPLC (eluents: $A=0.1 \%$ trifluoroacetic acid in water and $B=$ acetonitrile; gradient $0-25 \min 95-50 \%$ B) to determine the relative amount of intact peptide and its metabolites in the serum. The half-life was calculated fitting a first-order reaction to the experimental data (Equation 1) using Prism software (GraphPad Software Inc.).

\section{Saturation binding experiments}

DOTA-PEG4-BN conjugates were studied in vitro performing saturation binding experiments on whole cells. The DOTA coupled derivatives were labeled in Eppendorf tubes containing $10 \mu \mathrm{L}$ of ${ }^{111} \mathrm{InCl}_{3}(50-150 \mathrm{KBq} / \mu \mathrm{L})$, $1 \mathrm{nmol}$ of peptide (10 $\mu \mathrm{M}$ final concentration), and sodium acetate buffer $(0.4 \mathrm{M}, \mathrm{pH} 5)$ adjusted to a final volume of $100 \mu \mathrm{L}$. The solution was incubated for $30 \mathrm{~min}$ at $95^{\circ} \mathrm{C}$ in a heating block. Silica gel thin layer chromatography using $0.1 \mathrm{~N}$ sodium citrate $(\mathrm{pH} 5)$ as mobile phase was utilized for quality control. Radiochemical purity was $\geq 98 \%$. Saturation of free chelators with cold indium was not performed. Confluent PC-3 human prostate cancer cells were seeded in six-well plates $\left(\sim 1.0 \times 10^{6}\right.$ cells $) 24 \mathrm{~h}$ before starting the experiments. Increasing concentrations of the ${ }^{111}$ In-DOTA-peptide ranging from 50 to $0.05 \mathrm{nM}$ were assigned to different wells. For blocking experiments, excess of the respective unlabeled peptide was used. For each radioligand, triplicates were prepared for every concentration, for both total binding and nonspecific binding. Before adding the radioligands to the wells, the plates were placed on ice for $30 \mathrm{~min}$. After adding the radioligands and the blocking for nonspecific binding, the plates were incubated for $1 \mathrm{~h}$ at $4{ }^{\circ} \mathrm{C}$. Afterwards, the binding buffer was removed and the cells were washed twice with ice-cold phosphate-buffered saline (PBS; pH 7.4). Cell-

Table 1 Peptide conjugates with their corresponding peptide sequence, retention time $\left(R_{\mathrm{t}}\right)$, molecular weight (MW), and LC-MS data. All peptides contain DOTA-PEG4 on the N-terminus

\begin{tabular}{|c|c|c|c|c|}
\hline Peptide conjugates & Peptide sequence & $R_{\mathrm{t}} / \mathrm{min}$ & Mw/u.m.a. & {$\left[\mathrm{M}+2 \mathrm{H}^{+}\right] / 2$} \\
\hline BN1 & -Gln-Trp-Ala-Val-Gly-His-Leu-Met-NH & 12.91 & 1595 & 797.4 \\
\hline BN2 & -Gln-Trp-Ala-Val-Gly-His-Cha-Nle-NH 2 & 13.66 & 1630 & 817.6 \\
\hline BN3 & -Gln-Trp-Ala-Val-Gly-His-Cha-Nle-Glu-NH & 13.55 & 1759 & 880.7 \\
\hline BN4 & -Gln-Trp-Ala-Val-Gly-His-Sta-Leu-NH ${ }_{2}$ & 12.56 & 1636 & 819.3 \\
\hline BN5 & -GIn-Trp-Ala-Val-NMeGly-His-Sta-Leu-NH ${ }_{2}$ & 12.46 & 1649 & 825.4 \\
\hline BN6 & -DPhe-GIn-Trp-Ala-Val-Gly-His-Sta-Leu-NH ${ }_{2}$ & 13.19 & 1783 & 891.4 \\
\hline BN7 & -DPhe-Gln-Trp-Ala-Val-Gly-His-Cha-Nle-NH ${ }_{2}$ & 14.35 & 1780 & 891.5 \\
\hline BN8 & -DPhe-GIn-Trp-Ala-Val-NMeGly-His-Sta-Leu-NH 2 & 13.13 & 1798 & 900.3 \\
\hline
\end{tabular}


bound radioactivity was subsequently recovered by trypsinization of the wells. Radioactivity in the bound and free fractions was determined with a WIZARD gamma counter (Wallac, Turku, Finland). Specific binding was calculated by subtracting nonspecific from total binding at each concentration of radioligand. Affinity $\left(K_{\mathrm{d}}\right)$ and binding site density $\left(B_{\max }\right)$ were calculated from Scatchard plots using Prism software (GraphPad Software Inc.).

\section{Biodistribution and imaging experiments in nude mice bearing subcutaneous PC-3 xenografts}

For animal experiments, the institutional and national guide for the care and use of laboratory animals was followed. CD1 nude mice $(n=36$, female, 6 weeks old, $18-22 \mathrm{~g}$ body weight) were subcutaneously injected in the right thigh with $2 \times 10^{6} \mathrm{PC}-3$ cells in $300 \mu \mathrm{l}$ of a mediumto-matrigel solution (50:50, v:v, BD Biosciences). After tumor growth (approximately 20 days), mice were divided in four groups (BN1, BN4, BN7, and BN8). DOTA-PEG4$\mathrm{BN}$ conjugates were radiolabelled with ${ }^{111} \mathrm{In}$ as described elsewhere [26], and $1.85 \mathrm{MBq}(0.25 \mu \mathrm{g}, 7.4 \mathrm{MBq} / \mu \mathrm{g}$ in $100 \mu \mathrm{l} \mathrm{NaCl}$ ) of radiopharmaceutical was injected in the tail vein of each animal. Injected dose was determined by counting the syringe before and after injection. At $1 \mathrm{~h}$ post injection, three mice per group were anesthetized and placed on a horizontal support for planar imaging. The same procedure was repeated at $6 \mathrm{~h}$ and $24 \mathrm{~h}$ post injection. Images were acquired for 60 to $90 \mathrm{~s}$ with a highresolution scintigraphic camera [27] equipped with a square tungsten collimator integrated with a $\mathrm{CsI}(\mathrm{Tl})$ scintillation structure, coupled to a Hamamatsu H8500 Flat Panel Position Sensitive Photomultiplier Tube (PSPMT), a pure tungsten shielding housing, a charge readout electronics, and a data acquisition system for online image display. Region of interest (ROI) analysis was performed by drawing a region over the visible tumor (target) and copying it to the opposite thigh (background). The counts in the tumor ROI divided by the counts in the background ROI provided tumor-to-background (T/B) ratios. After each imaging session, mice were euthanized. The organs and the tumors were excised and weighed, and the radioactivity was determined using a single well $\gamma$-counter. Results were expressed as percentage of injected dose per gram $(\% \mathrm{ID} / \mathrm{g})$ of tissue.

\section{Results}

\section{Serum stability and saturation binding experiments}

Saturation binding experiments were performed on PC3 cells at $4{ }^{\circ} \mathrm{C}$. The results are summarized in Table 2 . With the exception of the BN3 derivative for which no specific binding was detectable, all compounds showed saturable binding with $K_{\mathrm{d}}$ in the order of $10^{-9} \mathrm{M}$ and $B_{\max }$ values in the order of $10^{5}$ receptor sites per cell.

Figure 2 shows a time course analysis of the stability of each derivative labeled with ${ }^{177} \mathrm{Lu}$ and incubated in human serum. The half-lives derived from fitting the experimental data are reported in Table 2. There were some clear differences among the tested compounds, indicating that the amino acid substitutions performed have important effect on compound stability.

As shown in Fig. 2, studied peptides could be grouped according to their half-life. In Fig. 2a, serum stabilities of the rapidly degraded peptides are reported: $\mathrm{BN} 1$, the native bombesin sequence that, as expected, showed very rapid degradation with a half-life value of $16.1 \mathrm{~h}$; BN3 (half-life of $40.7 \mathrm{~h}$ ), and the BN2 and BN7 peptides (showing half-lives around $20 \mathrm{~h}$ ) having the $\mathrm{Cha}^{13}-\mathrm{Nle}^{14}$ substitution and differing only for the $\mathrm{N}$-terminal $D P h e$. In Fig. $2 \mathrm{~b}$, the serum stability profiles of the peptides with the longer half-lives are shown (BN5, BN8, BN4, and BN6) with the first two showing half-life values over 15 days (354.2 and $414.1 \mathrm{~h}$, respectively).

\section{Biodistribution and imaging experiments in nude mice bearing subcutaneous PC-3 xenografts}

Figure 3 shows results of biodistribution experiments performed at 1,6 , and $24 \mathrm{~h}$ after injection of ${ }^{111}$ In labeled derivatives $\mathrm{BN} 1, \mathrm{BN} 4, \mathrm{BN} 7$, and $\mathrm{BN} 8$. The native

Table 2 Dissociation constants $\left(K_{\mathrm{d}}\right)$, apparent number of binding sites per cells $\left(B_{\text {max }}\right)$, and serum half-life of DOTA-PEG4-BN conjugates

\begin{tabular}{lllr}
\hline $\begin{array}{l}\text { Peptide } \\
\text { conjugates }\end{array}$ & $K_{\mathrm{d}} / \mathrm{nM}$ & $B_{\max }$ (binding sites/cell) ${ }^{*} 10^{5}$ & Half-life/h \\
\hline BN1 & $3.30 \pm 0.90$ & $2.45 \pm 0.73$ & 16.1 \\
BN2 & $4.27 \pm 1.28$ & $4.10 \pm 1.23$ & 20.9 \\
BN3 & $>1000$ & ND & 40.7 \\
BN4 & $4.97 \pm 1.40$ & $5.54 \pm 1.56$ & 224.1 \\
BN5 & $10.89 \pm 2.71$ & $1.56 \pm 0.39$ & 354.2 \\
BN6 & $3.90 \pm 1.15$ & $5.89 \pm 1.74$ & 208.3 \\
BN7 & $6.36 \pm 1.74$ & $5.32 \pm 1.46$ & 18.7 \\
BN8 & $5.91 \pm 1.77$ & $3.52 \pm 1.03$ & 414.1 \\
\hline
\end{tabular}



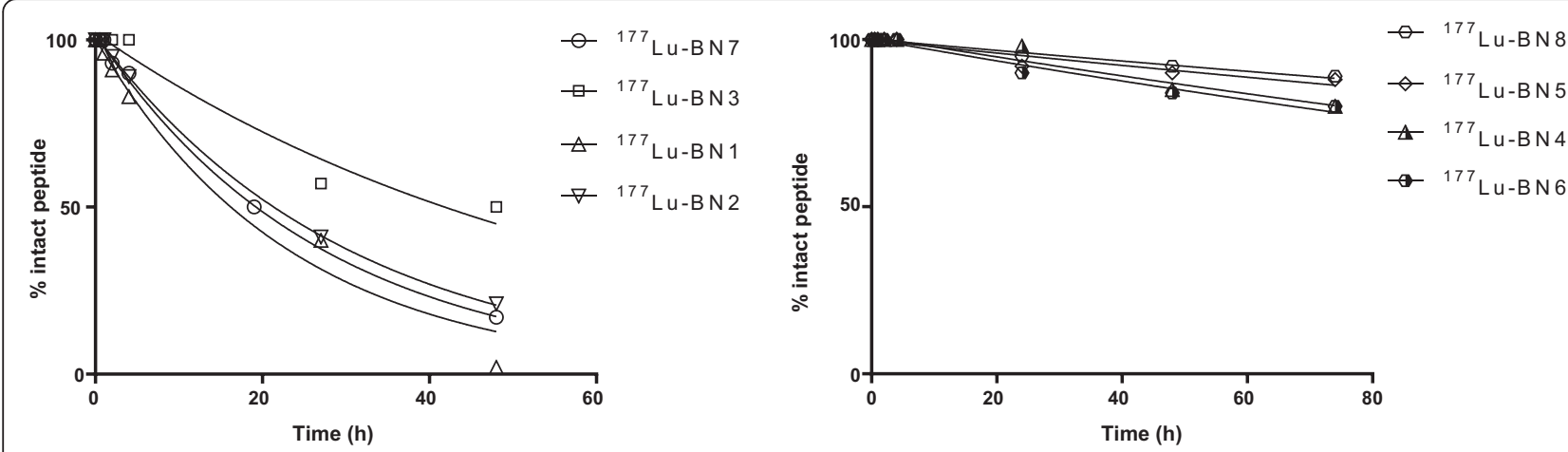

Fig. 2 In vitro serum stability of ${ }^{177} \mathrm{Lu}-\mathrm{BN}$ peptides at $37^{\circ} \mathrm{C}$

BN1 peptide shows very rapid blood clearance, and already at $1 \mathrm{~h}$ post injection, there is very low blood activity. High uptake is observed in the kidneys compared to other organs. There is high level, prolonged accumulation in the GRP-R-rich pancreas. The tumor-targeting capabilities appear to be rather poor. The BN4 peptide appears to have the longest circulating half-life of all tested compounds with blood \% $\mathrm{ID} / \mathrm{g}$ in the order of $5 \%$ at $1 \mathrm{~h}$. The compound shows some hepatobiliary clearance with high $\% \mathrm{ID} / g$ values in the intestines and prolonged high-level kidney retention. The receptorpositive PC-3 xenografts and pancreas showed very high initial uptake that dropped at later time points. Due to permanently high circulating activity, $\mathrm{T} / \mathrm{B}$ ratios calculated in vivo are the least favorable of all peptides. The BN7 peptide showed very rapid blood clearance, some hepatobiliary excretion, and overall poor targeting to the receptor-positive xenografts. The BN8 peptide, a compound that by design should have antagonistic activity, performed better than BN1 and BN7, with higher, at least at the first two time points, accumulation in the receptor-positive xenografts with low circulating activity and highest T/B ratio in vivo (Figs. 3 and 4).

Gamma camera experiments confirmed data obtained from organ biodistribution studies. Figure 5 shows images obtained $6 \mathrm{~h}$ after injection in representative mice
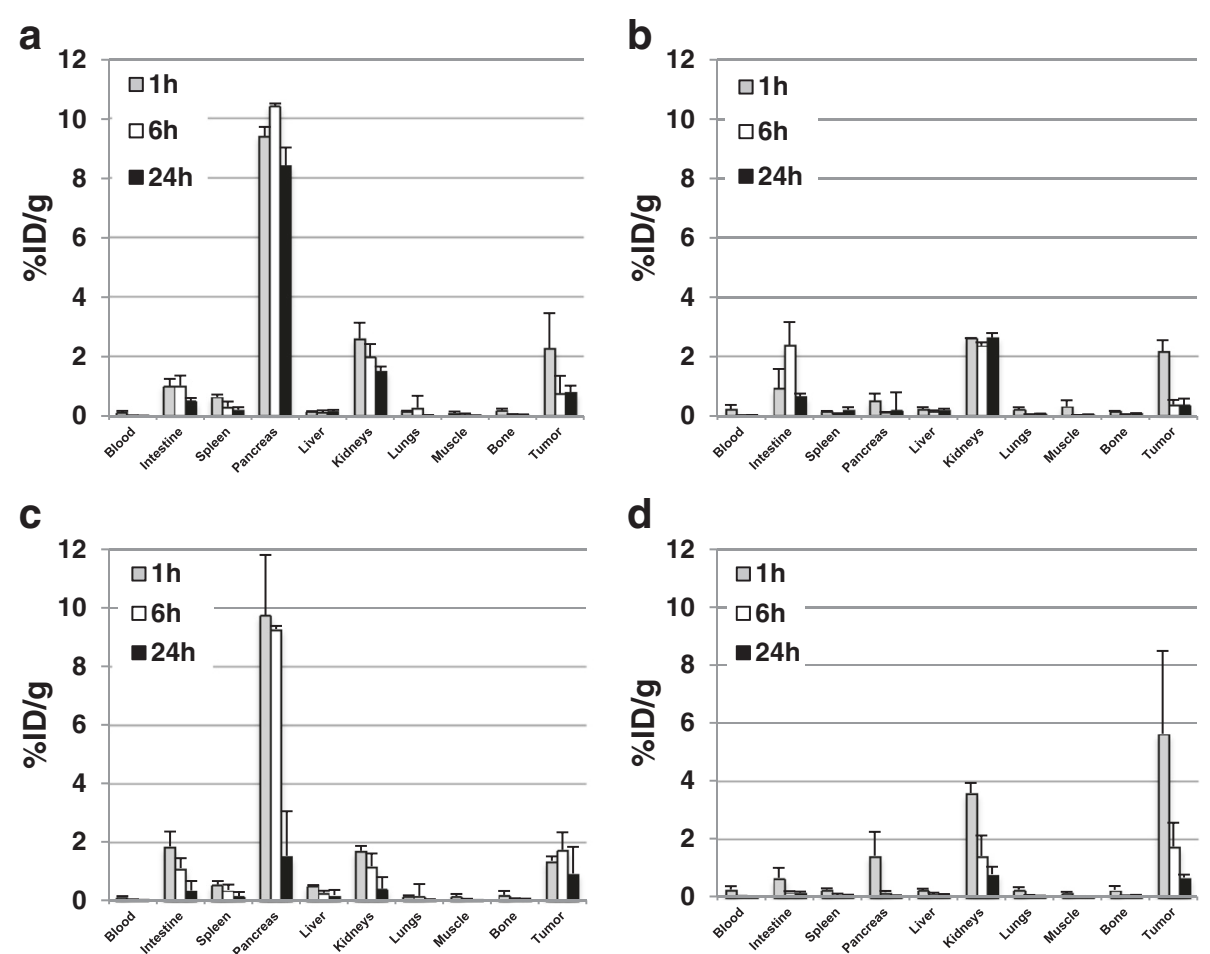

Fig. 3 Biodistribution of different radiolabeled bombesin derivatives. a BN1. b BN4. c BN7. d BN8. Data are expressed as \%ID/g, and error bars are standard deviations of three mice per time point. The highest tumor uptake with low pancreas, liver, and kidney uptake is BN8 

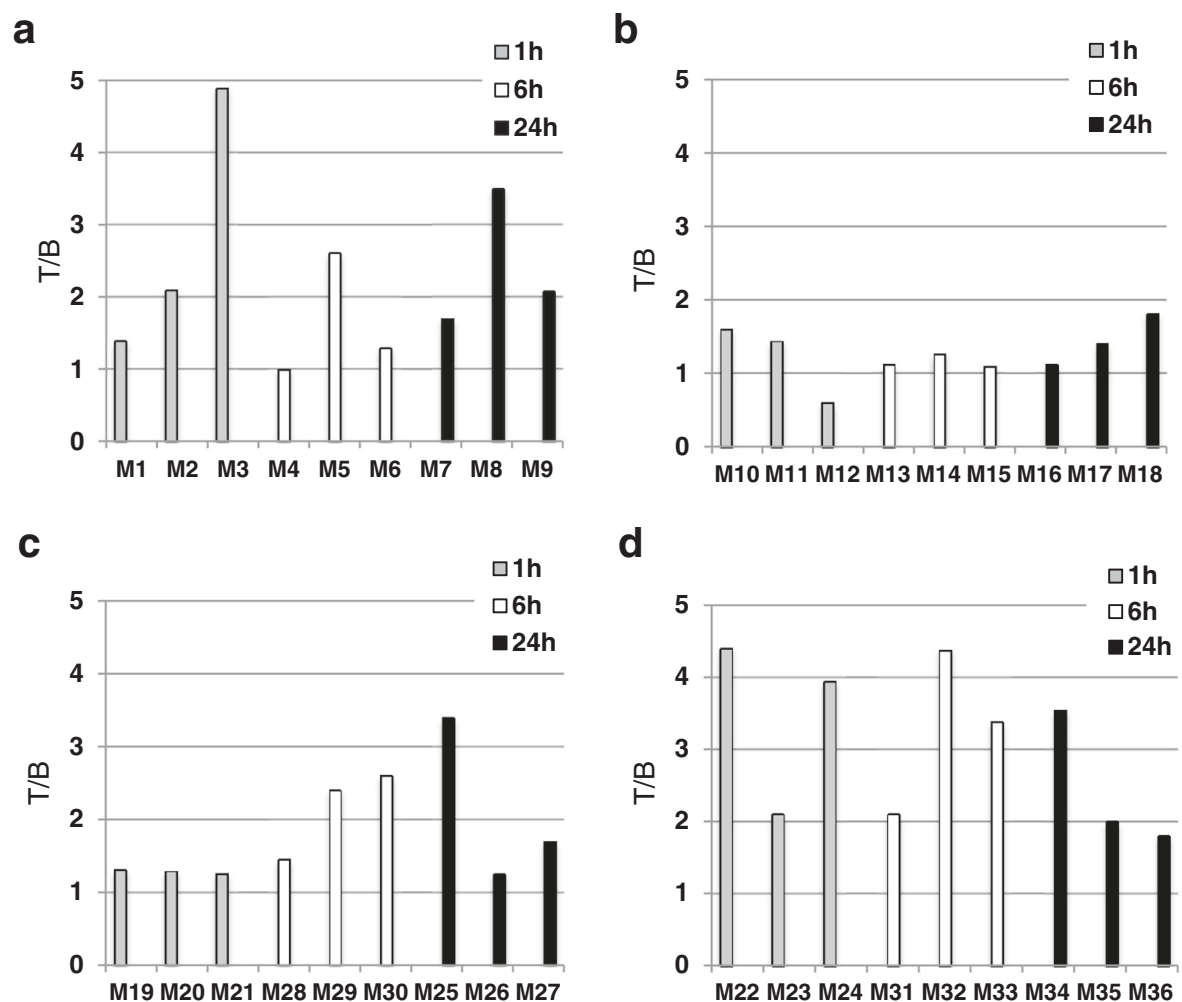

Fig. 4 Tumor-to-background (T/B) ratios in mice with PC-3 xenografts injected with different radiolabeled bombesin derivatives. a BN1. b BN4. c BN7. d BN8. The BN8 derivative shows the highest ratios among the compounds tested

from the four groups tested. Although the tumor is clearly visible with all compounds tested, the BN8 compound shows better imaging properties with higher contrast relative to surrounding tissue in the $\mathrm{PC}-3$ xenografts.

\section{Discussion}

Due to its high overexpression, GRP-R is an attractive target receptor for imaging and therapy of several cancers. Different peptides based on the wild-type BN [7-14] sequence, with agonist or antagonist properties, have been described in recent years and have been characterized as imaging tracers after their labeling with gamma or positron emitters. However, several different approaches for peptide labeling and other modifications, such as introduction of spacers between the targeting moiety and the chelator group, have been described. This does not allow to accurately compare and evaluate the importance of the peptide structure for binding and imaging properties. We have attempted to address this issue by comparing eight $\mathrm{BN}[7-14]$ peptide conjugates having the same spacer and metal chelator at $\mathrm{N}$-terminus.

Based on data available in the literature, we have selected and characterized eight DOTA coupled peptide sequences that are bombesin analogs (Fig. 1). These publications served as basis for peptide design
$[15,19,26,28,29]$. For the BN2 peptide, Leu ${ }^{13}$-Met $^{14}$ has been substituted with $\mathrm{Cha}^{13}-\mathrm{Nle}^{14}$, a previously described strategy aimed at improving peptide stability in vivo [13]. The BN3 analog derives from the BN2 analog and contains an additional glutamic acid residue on the $\mathrm{C}$-terminus that was introduced in an attempt to improve solubility. In BN4, the dipeptide Leu ${ }^{13}$-Met ${ }^{14}$ has been substituted with $\mathrm{Sta}^{13}$-Leu ${ }^{14}$ in an effort to improve in vivo stability to aminopeptidase activity. The Leu ${ }^{13}$-to-Sta ${ }^{13}$ substitution has also been shown to provide antagonistic properties to the peptide [17]. Peptide BN5 is a further modification of BN4 in which the Gly ${ }^{11}$ residue has been substituted with $\mathrm{N}$-methylglycine to improve stability of the GlyHis bond to carnosinase enzymatic cleavage in vivo. The BN6, BN7, and BN8 peptides are modifications of the BN4, BN2, and BN5 peptides, respectively, in which a $D P h e$ has been introduced at the $\mathrm{N}$-terminus in order to increase binding affinity as previously described [11]. A hydrophilic spacer, formed by four ethoxylic groups (PEG4) was interposed between the peptide and the DOTA chelator. This approach has been previously described with DOTA coupled bombesin derivatives, and this particular spacer length was chosen based on evidence showing improved pharmacokinetic properties [22]. Peptides 


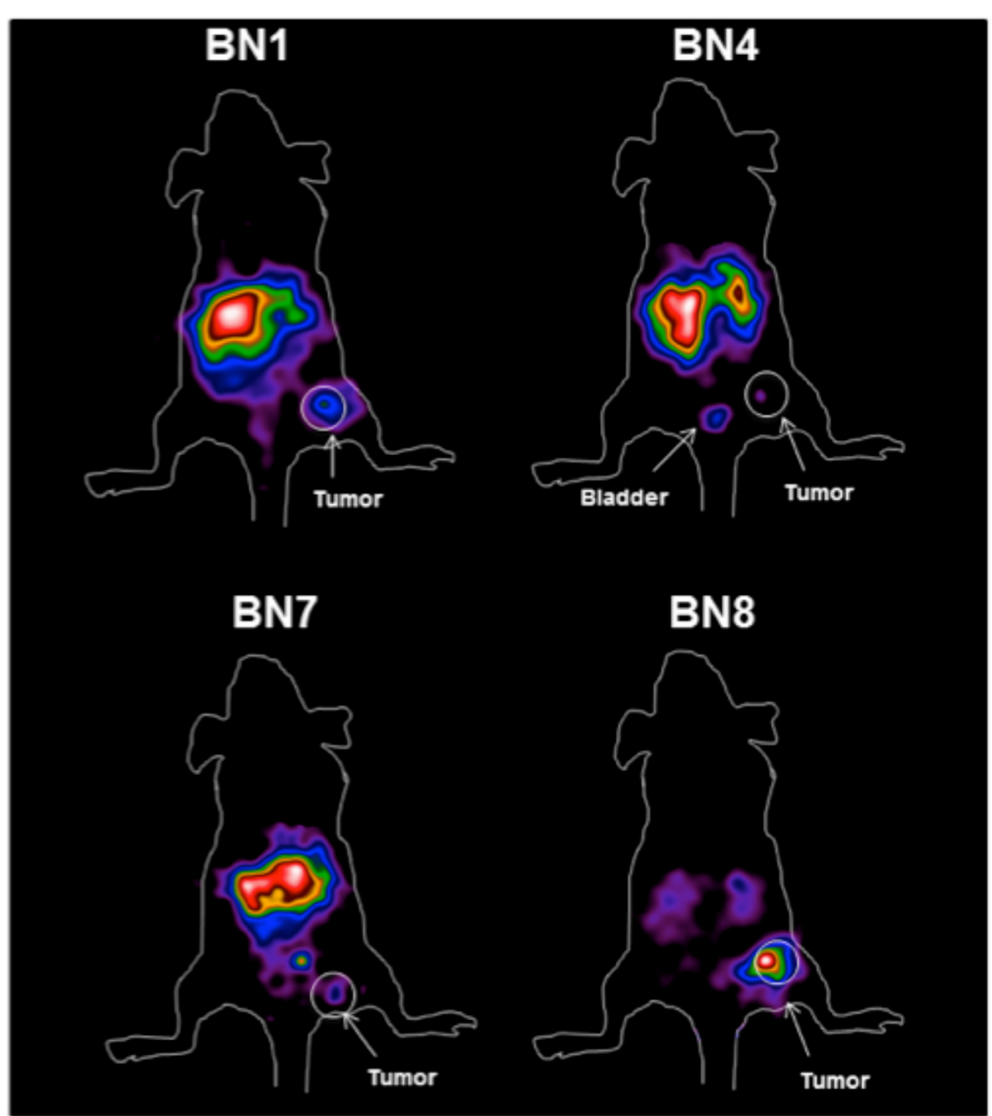

Fig. 5 Dorsal view gamma camera images obtained $6 \mathrm{~h}$ post injection. Subcutaneous tumors in the right thigh are indicated. The BN8 derivative shows the best contrast of tumor uptake compared to other organs (mice are M5, M14, M29, and M33)

were synthesized in solid phase with $\mathrm{Fmoc} / \mathrm{tBu}$ chemistry and obtained in high purity after RP-HPLC chromatography.

The radiolabeled peptide derivatives show no significant differences in affinity and binding capacity on PC-3 cells, with the exception of the BN3 derivative. This derivative has a modification on the $\mathrm{C}$-terminus that appears to completely block its ability to bind to the receptor. The dissociation constants $\left(K_{\mathrm{d}}\right)$ of the remaining seven ${ }^{111}$ In-labeled peptides are in the nanomolar range and are in agreement with previously published data on this same cell line with similar derivatives $[13,19,21]$. It has been previously reported [11] that the addition of a $D$ Phe residue at the $\mathrm{N}$-terminus would positively affect peptide-binding affinity; however, we did not observe any significant increase in affinity by comparing the DPhe coupled derivatives (BN6, BN7, and BN8) with their respective counterparts not containing DPhe (BN4, $\mathrm{BN} 2$, and BN5).

Serum stability was studied in human serum after labeling. Due to its weekly availability in our laboratory (University Hospital Freiburg) in high quantity, we chose ${ }^{177} \mathrm{Lu}$ for these experiments. There are some clear differences among the compounds tested, indicating that the amino acid substitutions within the binding sequence have important effect on peptide stability. The native $\mathrm{BN} 1$ sequence shows very rapid degradation. The fast degradation of the wild-type $\mathrm{BN}$ derivative (BN1) is in agreement with the behavior observed for ${ }^{177} \mathrm{Lu}$ AMBA [30] in which the same peptide sequence is modified at the $\mathrm{N}$-terminus with a 4-aminobenzoyl spacer and the DO3A chelator.

The BN8 and BN5 derivatives, the two peptides containing the NMeGly ${ }^{11}$ as well as the Sta ${ }^{13}$ substitutions, show very long plasma half-lives (over 15 days in both cases). The two compounds differ only for the N-terminal DPhe that appears not to affect plasma stability.

Similarly, the BN4/BN6 pair shows fairly long plasma half-life and, also in this case, the addition of the $\mathrm{N}$ terminal DPhe does not appear to have an impact on stability.

The BN2/BN7 pair having the $\mathrm{Cha}^{13}-\mathrm{Nle}^{14}$ substitution and again differing only for the N-terminal DPhe shows superimposable plasma half-lives and the rate of degradation is in agreement with previously reported data on the same peptide sequence labeled with ${ }^{99 m} \mathrm{Tc}$ 
using a different approaches [13]. These two peptides, however, show more rapid degradation compared to the BN5/BN8 and BN4/BN6 pairs that contain the Sta ${ }^{13}$ Leu ${ }^{14}$ substitutions.

The high affinity binding of the seven of the tested derivatives suggests they are all suitable for in vivo use as GRP-R targeting radiopharmaceuticals. We chose only four for further in vivo studies. The BN1 peptide served as historical reference as it is the one from which all other peptides are derived. Given the clear similarities in the in vitro properties of the BN4/BN6, BN2/BN7, and BN5/BN8 pairs, we chose one from each pair for the in vivo studies. We therefore characterized the DPhe-containing BN7 and BN8 peptides whereas the non-DPhecontaining BN4 was chosen over the BN6 peptide as the latter has been previously characterized in the same animal model [22]. In the interest of limiting the number of animals and given the fact that very low nonspecific retention in both the PC-3 xenografts and the receptorrich pancreas has been previously reported for similar DOTA coupled peptides [26], we deliberately chose not to perform blocking experiments.

The BN1 peptide showed very rapid blood clearance, very high prolonged uptake in the receptor-rich pancreas, and lower targeting of the PC-3 xenografts that was transient as it diminished over time. These biodistribution properties are very similar to those previously described for ${ }^{177}$ Lu-AMBA [30] that also shows prolonged specific retention in the pancreas, transient accumulation in receptor-positive tumor, and rapid blood clearance. The remaining three peptides, BN4, BN7, and BN8, show some similar pattern in biodistribution properties.

All three show transient high accumulation in the PC3 tumor and pancreas that drops over time. Although tumor accumulation rapidly decreases over time, there is still a fairly wide time window where high target-tobackground ratios were observed. The missing DPhe residue in BN4 may be responsible for the poor pharmacokinetic performance of this analog compared to that reported for the $D$ Phe derivative [22]. The uptake in tumor and receptor-positive organs such as the pancreas is reasonably high at the early time point but rapidly decreases over time. The biodistribution profile of the HisCha derivative (BN7) mirrors the one reported previously on a similar molecule with high uptake in the pancreas at the early time point that decreases over time and with overall fairly low tumor uptake [21]. A favorable pharmacokinetic profile is shown by BN8 with high tumor uptake and good tumor-to-non-target-tissue ratios already at $1 \mathrm{~h}$. This analog is the only one showing the highest uptake in the xenograft compared to all other organs at all times tested. Given this feature and the advantageous target-tobackground ratios observed, this derivative seems to have the best characteristics among the eight compounds tested, particularly for imaging applications using short half-lived isotopes such as ${ }^{68} \mathrm{Ga}$.

\section{Conclusions}

We have performed a side-by-side comparison of eight different peptide sequences targeting the GRP-R. Our data indicate that among the peptides tested, the BN5/ BN8 pair with the NMeGly ${ }^{11}$ and $\mathrm{Sta}^{13}$-Leu ${ }^{14}$ substitutions would be the most suitable compound for rapid high-contrast GRP-R targeting for imaging applications in vivo.

\section{Competing interests}

The authors declare they have no competing interests.

\section{Authors' contributions}

$A A, F G, R M, A S, G M$, and $L A$ are involved in the study design. AA, FG, RM, LDP, MA, AM, PR, AS, GM, and AL performed the experiments. AA, FG, RM, $A S, G M$, and $A L$ prepared the manuscript. All authors read and approved the final manuscript.

\section{Acknowledgements}

The authors wish to thank Dr. A. Soluri and R. Massari, IBB-CNR, Montelibretti (RM), Italy, for providing the high-resolution scintigraphic camera for mouse imaging. This research was funded by Regione Campania under POR Campania FESR 2007-2013 - 0.O. 2.1 (FarmaBioNet) and by the Italian Ministry for Research (M.I.U.R.), 0016/09 593/2000 (Invectors) project

\section{Author details}

"Department of Pharmacy, CIRPeB, University of Naples "Federico II" and Invectors srl, Napoli, Italy. ${ }^{2}$ Nuclear Medicine Unit, Department of Medical-Surgical Sciences and of Translational Medicine, Faculty of Medicine and Psychology, "Sapienza" University of Roma, Rome, Italy. ${ }^{3}$ Department of Nuclear Medicine, University Hospital Freiburg, Freiburg, Germany. ${ }^{4}$ Centro Ricerche Oncologiche Mercogliano, Istituto Nazionale Tumori "Fondazione G. Pascale"-IRCCS, Mercogliano (AV), Italy. ${ }^{5}$ Struttura Complessa Medicina Nucleare, Istituto Nazionale Tumori "Fondazione G. Pascale"-IRCCS, Via M. Semmola, 52, Napoli 80131, Italy.

Received: 4 December 2015 Accepted: 15 February 2016

Published online: 20 February 2016

References

1. Majumdar ID, Weber HC. Biology of mammalian bombesin-like peptides and their receptors. Curr Opin Endocrinol Diabetes Obes. 2011;18(1):68-74. doi:10.1097/MED.0b013e328340ff93.

2. Reubi JC. Somatostatin and other peptide receptors as tools for tumor diagnosis and treatment. Neuroendocrinology. 2004;80 Suppl 1:51-6. doi:10. $1159 / 000080742$.

3. Kahkonen E, Jambor I, Kemppainen J, Lehtio K, Gronroos TJ, Kuisma A, et al. In vivo imaging of prostate cancer using [68Ga]-labeled bombesin analog BAY86-7548. Clin Cancer Res. 2013;19(19):5434-43. doi:10.1158/1078-0432. CCR-12-3490.

4. Wieser G, Mansi R, Grosu AL, Schultze-Seemann W, Dumont-Walter RA Meyer PT, et al. Positron emission tomography (PET) imaging of prostate cancer with a gastrin releasing peptide receptor antagonist-from mice to men. Theranostics. 2014;4(4):412-9. doi:10.7150/thno.7324.

5. Lantry LE, Cappelletti E, Maddalena ME, Fox JS, Feng W, Chen J, et al. 177Lu-AMBA: Synthesis and characterization of a selective 177Lu-labeled GRP-R agonist for systemic radiotherapy of prostate cancer. J Nucl Med. 2006:47(7):1144-52

6. Bodei L, Ferrari M, Nunn A, Llull J, Cremonesi M, Martano L, et al. Lu-177AMBA bombesin analogue in hormone refractory prostate cancer patients: a phase I escalation study with single-cycle administrations. Eur J Nucl Med Mol Imaging. 2007:34:S221.

7. Cescato R, Maina T, Nock B, Nikolopoulou A, Charalambidis D, Piccand V, et al. Bombesin receptor antagonists may be preferable to agonists for tumor targeting. J Nucl Med. 2008;49(2):318-26. doi:10.2967/jnumed.107.045054. 
8. Broccardo M, Falconieri Erspamer G, Melchiorri P, Negri L, de Castiglione R. Relative potency of bombesin-like peptides. $\mathrm{Br}$ J Pharmacol. 1975;55(2):221-7.

9. Jensen RT, Battey JF, Spindel ER, Benya RV. International Union of Pharmacology. LXVIII. Mammalian bombesin receptors: nomenclature, distribution, pharmacology, signaling, and functions in normal and disease states. Pharmacol Rev. 2008;60(1):1-42. doi:10.1124/pr.107.07108.

10. Katsuno T, Pradhan TK, Ryan RR, Mantey SA, Hou W, Donohue PJ, et al. Pharmacology and cell biology of the bombesin receptor subtype 4 (BB4-R). Biochemistry. 1999;38(22):7307-20. doi:10.1021/bi990204w.

11. Mantey SA, Weber HC, Sainz E, Akeson M, Ryan RR, Pradhan TK, et al. Discovery of a high affinity radioligand for the human orphan receptor, bombesin receptor subtype 3 , which demonstrates that it has a unique pharmacology compared with other mammalian bombesin receptors. J Biol Chem. 1997;272(41):26062-71.

12. Pradhan TK, Katsuno T, Taylor JE, Kim SH, Ryan RR, Mantey SA, et al. Identification of a unique ligand which has high affinity for all four bombesin receptor subtypes. Eur J Pharmacol. 1998;343(2-3):275-87.

13. Garcia Garayoa E, Schweinsberg C, Maes V, Ruegg D, Blanc A, Blauenstein P, et al. New [99mTc]bombesin analogues with improved biodistribution for targeting gastrin releasing-peptide receptor-positive tumors. Q J Nucl Med Mol Imaging. 2007:51(1):42-50.

14. Shipp MA, Tarr GE, Chen CY, Switzer SN, Hersh LB, Stein H, et al. CD10/ neutral endopeptidase 24.11 hydrolyzes bombesin-like peptides and regulates the growth of small cell carcinomas of the lung. Proc Natl Acad Sci U S A. 1991;88(23):10662-6.

15. Hohne A, Mu L, Honer M, Schubiger PA, Ametamey SM, Graham K, et al. Synthesis, 18F-labeling, and in vitro and in vivo studies of bombesin peptides modified with silicon-based building blocks. Bioconjug Chem. 2008;19(9):1871-9. doi:10.1021/bc800157h.

16. Teufel M, Saudek V, Ledig JP, Bernhardt A, Boularand S, Carreau A, et al. Sequence identification and characterization of human carnosinase and a closely related non-specific dipeptidase. J Biol Chem. 2003;278(8):6521-31. doi:10.1074/jbc.M209764200.

17. Tokita K, Katsuno T, Hocart SJ, Coy DH, Llinares M, Martinez J, et al. Molecular basis for selectivity of high affinity peptide antagonists for the gastrin-releasing peptide receptor. J Biol Chem. 2001;276(39):36652-63. doi: 10.1074/jbc.M104566200

18. Liu Y, Hu X, Liu H, Bu L, Ma X, Cheng K, et al. A comparative study of radiolabeled bombesin analogs for the PET imaging of prostate cancer. J Nucl Med. 2013;54(12):2132-8. doi:10.2967/jnumed.113.121533.

19. Mansi R, Wang $X$, Forrer F, Kneifel $S$, Tamma ML, Waser B, et al. Evaluation of a 1,4,7,10-tetraazacyclododecane-1,4,7,10-tetraacetic acid-conjugated bombesin-based radioantagonist for the labeling with single-photon emission computed tomography, positron emission tomography, and therapeutic radionuclides. Clin Cancer Res. 2009;15(16):5240-9. doi:10.1158/ 1078-0432.CCR-08-3145.

20. Abiraj K, Mansi R, Tamma ML, Forrer F, Cescato R, Reubi JC, et al. Tetraamine-derived bifunctional chelators for technetium-99m labelling: synthesis, bioconjugation and evaluation as targeted SPECT imaging probes for GRP-receptor-positive tumours. Chemistry. 2010;16(7):2115-24. doi:10. 1002/chem.200902011.

21. Dapp S, Muller C, Garayoa EG, Blauenstein P, Maes V, Brans L, et al. PEGylation, increasing specific activity and multiple dosing as strategies to improve the risk-benefit profile of targeted radionuclide therapy with 177Lu-DOTA-bombesin analogues. EJNMMI Res. 2012;2(1):24. doi:10. 1186/2191-219X-2-24.

22. Jamous M, Tamma ML, Gourni E, Waser B, Reubi JC, Maecke HR, et al. PEG spacers of different length influence the biological profile of bombesinbased radiolabeled antagonists. Nucl Med Biol. 2014;41(6):464-70. doi:10. 1016/j.nucmedbio.2014.03.014.

23. Rogers BE, Manna DD, Safavy A. In vitro and in vivo evaluation of a 64Culabeled polyethylene glycol-bombesin conjugate. Cancer Biother Radiopharm. 2004;19(1):25-34. doi:10.1089/108497804773391649.

24. Chen X, Park R, Hou Y, Tohme M, Shahinian AH, Bading JR, et al. microPET and autoradiographic imaging of GRP receptor expression with 64CuDOTA-[Lys3]bombesin in human prostate adenocarcinoma xenografts. J Nucl Med. 2004;45(8):1390-7.

25. Accardo A, Mansi R, Morisco A, Mangiapia G, Paduano L, Tesauro D, et al. Peptide modified nanocarriers for selective targeting of bombesin receptors. Molecular bioSystems. 2010;6(5):878-87. doi:10.1039/b923147a.
26. Mansi R, Wang X, Forrer F, Waser B, Cescato R, Graham K, et al. Development of a potent DOTA-conjugated bombesin antagonist for targeting GRPr-positive tumours. Eur J Nucl Med Mol Imaging. 2011;38(1): 97-107. doi:10.1007/s00259-010-1596-9.

27. Soluri A, Atzeni G, Ucci A, Bellone T, Cusanno F, Rodilossi G et al. New device based on the super spatial resolution (SSR) method. Nuclear nstruments and Methods in Physics Research Section A: Accelerators, Spectrometers, Detectors and Associated Equipment. 2013;728:150-6. doi: http://dx.doi.org/10.1016/j.nima.2013.06.094.

28. Llinares M, Devin C, Chaloin O, Azay J, Noel-Artis AM, Bernad N, et al. Syntheses and biological activities of potent bombesin receptor antagonists. J Pept Res. 1999;53(3):275-83.

29. Abiraj K, Mansi R, Tamma ML, Fani M, Forrer F, Nicolas G, et al. Bombesin antagonist-based radioligands for translational nuclear imaging of gastrinreleasing peptide receptor-positive tumors. J Nucl Med. 2011;52(12):1970-8. doi:10.2967/jnumed.111.094375.

30. Linder KE, Metcalfe E, Arunachalam T, Chen J, Eaton SM, Feng W, et al. In vitro and in vivo metabolism of Lu-AMBA, a GRP-receptor binding compound, and the synthesis and characterization of its metabolites. Bioconjug Chem. 2009;20(6):1171-8. doi:10.1021/bc9000189.

\section{Submit your manuscript to a SpringerOpen ${ }^{\circ}$ journal and benefit from:}

- Convenient online submission

- Rigorous peer review

- Immediate publication on acceptance

- Open access: articles freely available online

- High visibility within the field

- Retaining the copyright to your article

Submit your next manuscript at $>$ springeropen.com 